\title{
Pressure balance between midplane and inner and outer divertor in ASDEX Upgrade H-mode discharges
}

\author{
A. Kallenbach, R. Dux, T. Eich, R. Fischer, A. Herrmann, B. Kurzan, H.W. Müller, R. Neu, \\ R. Pugno, M. Wischmeier, E. Wolfrum and the ASDEX Upgrade Team \\ Max-Planck-Institut für Plasmaphysik, IPP-EURATOM Association, \\ D-85748 Garching, Germany
}

\begin{abstract}
Electron pressure profiles have been obtained in the outer midplane and along the divertor target using various diagnostics in ASDEX Upgrade. Inter-ELM and ELM phases of $\mathrm{H}$ mode discharges with different power- and neutral fluxes in the divertor are investigated. To check the parallel pressure balance, mapping using equilibrium reconstruction is corrected/checked by physics constraints, like the upstream separatrix temperature according to power balance. Good agreement between the midplane pressure and the divertor pressure obtained from Langmuir probe measurements, assuming a Mach flow $=1$ into the sheath, is observed for the outer scrape-off layer (SOL). A pressure reduction around the strike point by more than one order of magnitude is observed for low power, high neutral flux conditions. Strong detachment/pressure loss is seen in the inner divertor for all conditions. During ELMs, higher electron pressures are obtained in the outer divertor SOL compared to the midplane.
\end{abstract}

Key words:

PACS: 52.55.Fa, 52.55.Rk, 52.25.Fi

JNM keyword codes: P0600

PSI-18 keywords: ASDEX Upgrade, Edge plasma, Divertor 
${ }^{*}$ Corresponding author address: Boltzmannstr. 2, D-85748 Garching, Germany

*Corresponding author email: Arne.Kallenbach@ipp.mpg.de

${ }^{*}$ Corresponding author phone: +49-89-3299 1721

${ }^{*}$ Corresponding author fax: +49-89-3299 1812

\section{Introduction}

The reduction of the plasma pressure in front of the divertor targets is an important ingredient to obtain broad power load profiles which are mandatory for reactor-grade power flux values. In the standard divertor model, charge exchange $(\mathrm{CX})$ and recombination provide the required momentum loss leading to divertor detachment and a considerable reduction of peak power flux densities. In experiments as well as in detailed edge modelling, conditions may vary from almost vanishing electron pressure in front of a target (fully detached state) up to almost identical pressure at midplane and target sheath entrance locations. While the total pressure is conserved in the absence of sources and sinks, the static pressure $\mathrm{p}_{e}=\mathrm{n}_{e} \cdot \mathrm{T}_{e}$ is expected to be reduced at the target sheath entrance by a factor 2 under the assumption of a Mach flow = 1 into the sheath region. Recent modelling of the detachment process for increasing upstream densities in Ohmic discharges with the SOLPS code package revealed pronounced discrepancies between the measured and predicted pressure evolution along the targets [1] if the experimental power flux and separatrix density have been used as input to the calculations. With its excellent edge and SOL diagnostic suite, ASDEX Upgrade is well suited to investigate the pressure balance between midplane and divertor targets. Apart from accurate measurements required in real space, flux surface mapping is required to connect corresponding measurements in midplane and divertor. To improve the accuracy of the 
equilibrium reconstruction, physics models are required to mark the relative positions of diagnostics and the separatrix upstream and downstream. This paper presents the experimental characterisation of divertor and midplane electron pressure profiles for ELMy H-mode conditions in ASDEX Upgrade with fully tungsten covered plasma facing components. Target profiles are obtained from Langmuir probe measurements, using strike point sweeps to allow the reconstruction of continuous spatial profiles [2]. The electron pressure at the target is compared to the corresponding values at the outer midplane obtained by combined Lithium beam and interferometer measurements and Thomson scattering for conditions with different divertor collisionalities.

\section{Evaluation of midplane electron pressure profiles}

Figure 1 shows detailed profiles in the outer midplane for a discharge in a series with different heating powers and neutral divertor fluxes, resulting in a variation of the degree of detachment / divertor collisionality. Density profiles are spline fits obtained from integrated data analysis (IDA) [3] combining Lithium beam emission profiles from the SOL up to the pedestal top and line integrated densities from DCN interferometry with $0.2 \mathrm{~ms}$ temporal resolution. Electron temperature profiles are parametrised as modified tanh fits [4] to Thomson scattering data, taken from identical discharges with edge and core settings of the TS system. Unless in cut-off, ECE data are taken from the center to $\rho_{p o l}=0.95$ to avoid nonthermal emission from the pedestal region. While IDA and ECE data are taken with their original spatial positions, the TS data have been radially shifted by typically $1 \mathrm{~cm}$ to obtain a fitted inter-ELM separatrix temperature of $100 \mathrm{eV}$ [5]. 


\section{Divertor profiles}

Profiles for $\mathrm{T}_{e}$ and $\mathrm{n}_{e}$ are obtained from Langmuir probes using strike point sweeps to obtain continuous spatial profiles with fixed probe locations. Since accurate equilibrium mapping with respect to the outer midplane position is essential to obtain pressure balance along field lines, the position of the separatrix strike point on the target is assigned by the sign change of the floating potential or target electric currents, which is expected to occur very close to the strike point due to the effect of Pfirsch-Schlüter currents for attached conditions [6]. Despite the fact that the physics of the target currents is expected to be more complex during ELMs, we use the experimentally well pronounced sign change of $\mathrm{V}_{\text {float }}$ during the ELM-related strike point movement as separatrix marker. An example is shown in figure 2 for the case with the maximum position correction. The obtained corrections for the strike point coordinate at the target, $\mathrm{ds}_{s e p}$ vary between 0 and $15 \mathrm{~mm}$ for the discharges used, resulting in radial profile shifts up to $2 \mathrm{~mm}$ in the outer midplane. Since no strict model is available to predict the exact $\mathrm{V}_{\text {float }}$ sign location, the overall uncertainty of the separatrix position is assumed to be \pm 10 $\mathrm{mm}$ along the target, corresponding to less than $\pm 2 \mathrm{~mm}$ in the outer midplane.

\section{Electron pressure balance}

Figure 3 shows midplane and divertor profiles of $\mathrm{T}_{e}$ and $\mathrm{n}_{e}$ for 4 discharges with different divertor neutral densities, $\mathrm{n}_{0, \text { div }}$, obtained from a pressure gauge and different power fluxes to the divertor $\left(\mathrm{P}_{d i v}=\mathrm{P}_{\text {heat }}-\mathrm{P}_{\text {rad }, t o t}\right)$. While the divertor $\mathrm{T}_{e}$ profiles are quite flat, the $\mathrm{n}_{e}$ profiles exhibit broadening and a shift towards the SOL region with decreasing power per neutral, $\mathrm{P}_{d i v} / \mathrm{n}_{0, d i v}$. Also shown are the ratios of the electron density and temperature gradient lengths, $\eta_{e}$, around the midplane separatrix. The values $\eta_{e} \approx 2$ as reported earlier using TS density 
data [7] are again observed in the region of the edge transport barrier. The data of figure 3 allow a direct comparison of midplane and divertor pressures, as shown in figure 4 . The total pressures are estimated by the static pressure measured upstream (neglecting a possible flow) and by the divertor pressure multiplied by the factor 2, assuming a Mach flow $=1$ towards the target at the position of the Langmuir probe measurement at the sheath entrance. In between ELMs, very good agreement between the target and midplane pressure is observed through the outer SOL. Around the separatrix, a pronounced pressure drop occurs for lower values of $\mathrm{P}_{d i v} / \mathrm{n}_{0, d i v}$. Similar to the Ohmic cases, [8], modelling with the SOLPS code package fails to reproduce this pressure drop around the strike point when the experimental power- and neutral fluxes are used as input.

The inner target is almost completely detached with strike point pressures reduced by more than two orders of magnitude around the strike point. The inner divertor pressure shown in figure 4 is taken from a similar pulse of the previous campaign. Due to recent modifications in target and probe design, the evaluation of $\mathrm{T}_{e}$ is not possible for the current discharges due to too flat $\left(<1^{o}\right)$ field line angles of incidence here. Measurements of $\mathrm{j}_{\text {sat }}$, which are reliable for both campaigns indicate indeed similar detachment conditions in the inner divertor. The relative pressure drop decreases towards the SOL with increasing distance from the inner strike point, but is always far more pronounced than in the outer divertor.

During ELMs, the interpretation of the diagnostics, in particular of the Langmuir probes, is more uncertain. Strong fluctuations of the plasma potential in front of the target are observed, which occasionally exceed the triple probe bias voltage over a toroidal distance of $0.1 \mathrm{~m}$ and lead to negative spikes in the ' $\mathrm{j}_{\text {sat }}$ ' measurement. Reduction of the statistical contribution of the errors by coherent averaging of a large number of ELMs reveals approximate pressure balance (assuming Mach flow =1) between outer target close to the separatrix, while higher 
electron pressures are obtained for the far SOL divertor region. Inspection of the $\mathrm{T}_{e}$ and $\mathrm{n}_{e}$ profiles during the ELM shows that the increase of pressure towards the divertor SOL is due to a rise in density. No explanation of this apparent (transient) pressure imbalance can be given currently. Possible reasons are the effect of hot filaments which are not properly represented by the time-averaged Thomson scattering data or strong neutral ionisation due to the ELM power flux in the divertor. Time-dependent modelling, possibly including kinetic effects, will be required.

\section{Conclusions}

Close pressure balance is observed between midplane and outer divertor SOL in ASDEX Upgrade H-mode discharges. Reduced pressures are observed generally in the inner divertor and in the outer strike point region for low power fluxes into the divertor and high neutral densities. The corresponding pressure loss mechanism is still unclear, modelling with SOLPS does not reproduce a significant pressure drop when the experimental boundary conditions are used. During ELMs, transiently higher values of $\mathrm{p}_{e}$ are observed in the outer divertor SOL compared to upstream profiles.

\section{References}

[1] M. Wischmeier, X. Bonnin, D. Coster, A. Chankin, A. Kallenbach, et al., Contrib. Plasma Phys. 48 (2008) 249.

[2] A. Kallenbach, R. Dux, T. Eich, R. Fischer, L. Giannone, et al., Nucl. Fusion 48 (2008) 085008.

[3] R. Fischer, E. Wolfrum, J. Schweinzer, and the ASDEX Upgrade Team, Plasma Phys. Controlled Fusion 50 (2008) 085009. 
[4] A. Kallenbach, R. Dux, J. Gafert, G. Haas, L. D. Horton, et al., Nucl. Fusion 43 (2003) 573.

[5] A. Kallenbach, N. Asakura, A. Kirk, A. Korotkov, M. A. Mahdavi, et al., J. Nucl. Mater. 337-339 (2005) 381 .

[6] M. Schaffer, A. Chankin, H. Guo, G. Matthews, and R. Monk, Nucl. Fusion 37 (1997) 83.

[7] J. Neuhauser, D. Coster, H. U. Fahrbach, J. C. Fuchs, G. Haas, et al., Plasma Phys. Controlled Fusion 44 (2002) 855.

[8] M. Wischmeier et al., O-25, this conference . 

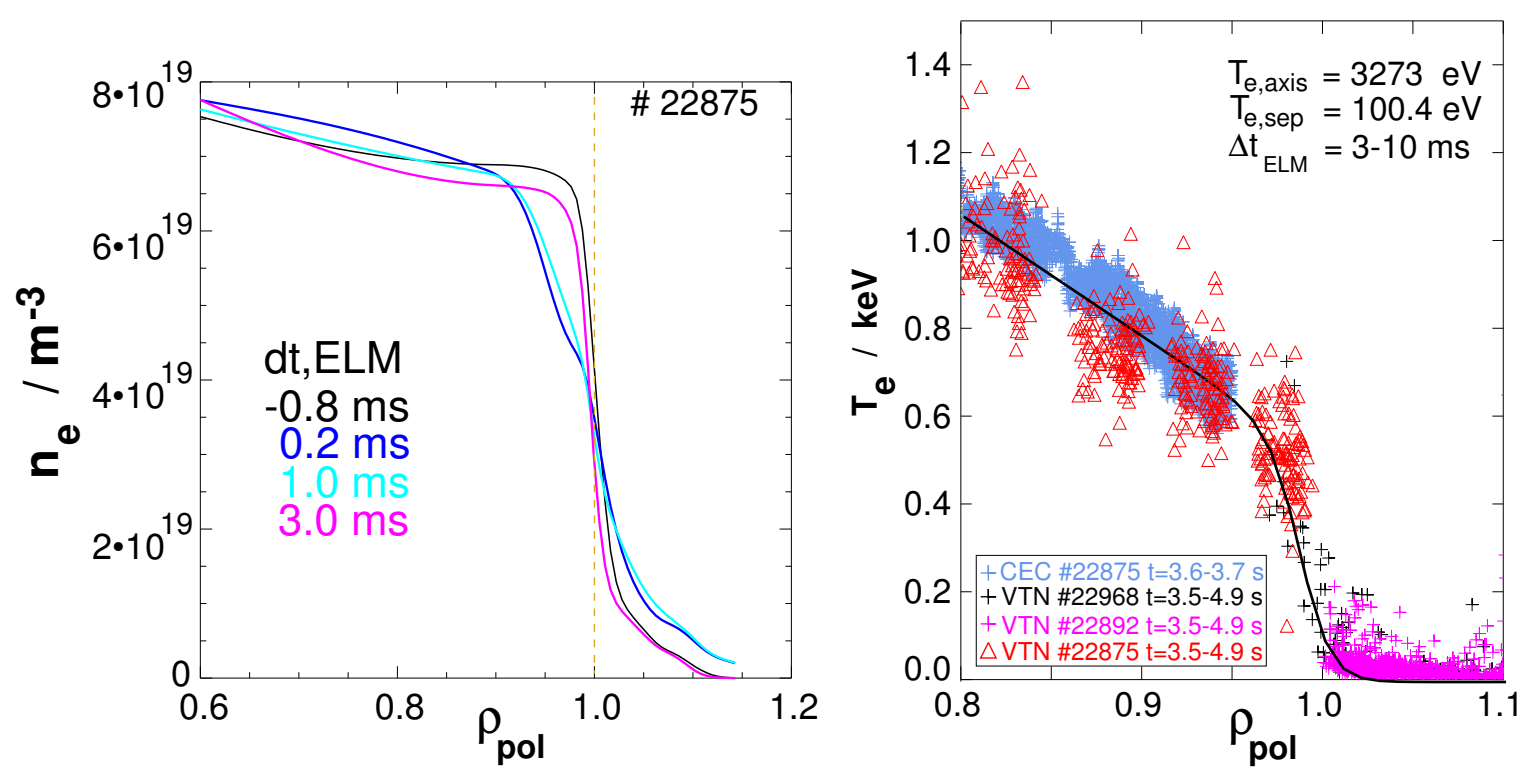

Fig. 1. Midplane profiles of electron density and temperature. Electron density profiles are obtained from IDA analysis of Li-beam and interferometer measurements. Different profiles show the evolution over the ELM cycle, obtained by coherent averaging of 150 ELMs. Electron temperatures are parametrized as modified tanh fits using ECE data and Thomson scattering (TS) data, here from 3 identical discharges with different TS and plasma positions taken during inter-ELM times. The $\mathrm{T}_{e}$ values in the outer SOL are uncertain due to the decreasing $\mathrm{S} / \mathrm{N}$ ratio of the TS diagnostic. 

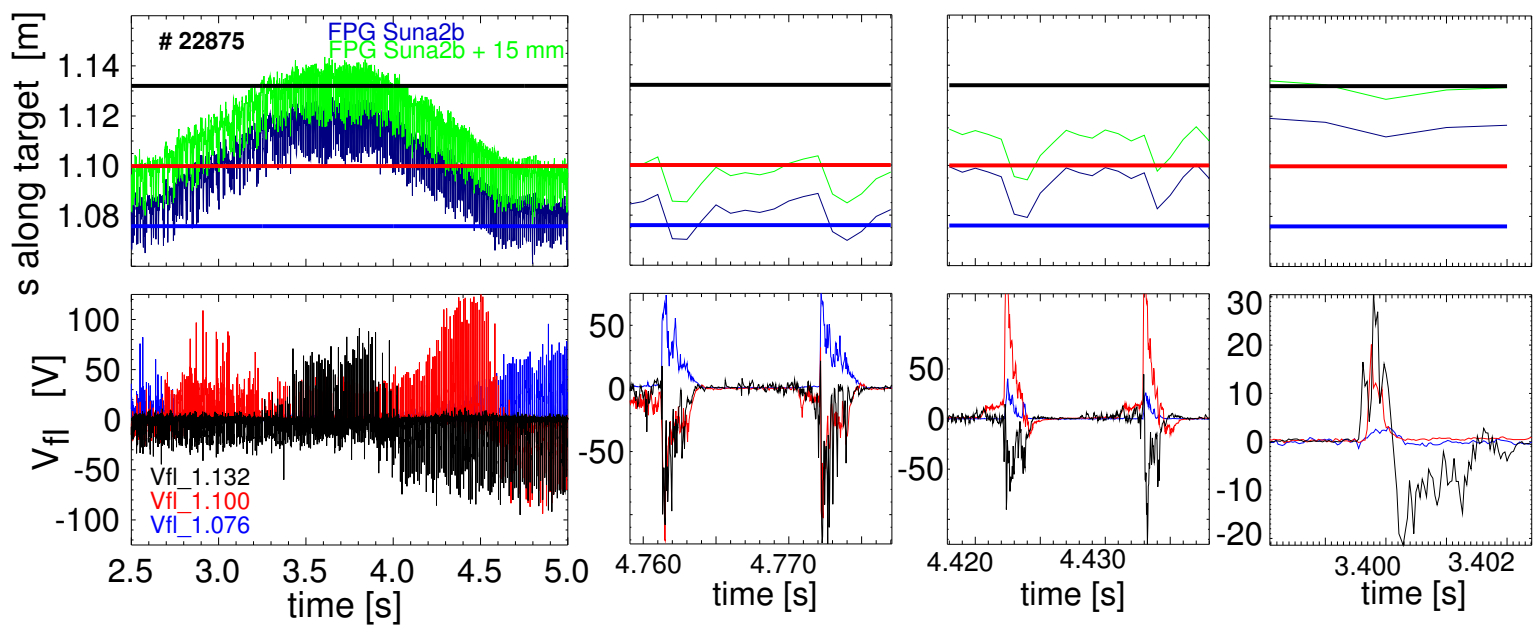

Fig. 2. Floating potentials during strike point sweeps at 3 different probe locations in the outer divertor, denoted by the s-coordinate measured in poloidal direction along the target. The positions of the 3 probes are indicated by horizontal lines. When the strike point crosses a probe, a sign change of the floating potential from negative (probe in SOL) to positive (probe in PFR) values is expected. The dynamic downward (i.e. to lower s-coordinate) movement of the strike point during an ELM produces a transient sign change. The assumption of a sign change of $\mathrm{V}_{\text {float }}$ at the strike point suggests the strike point to be $15 \mathrm{~mm}$ above the value obtained from fast equilibrium reconstruction by function parametrization. 

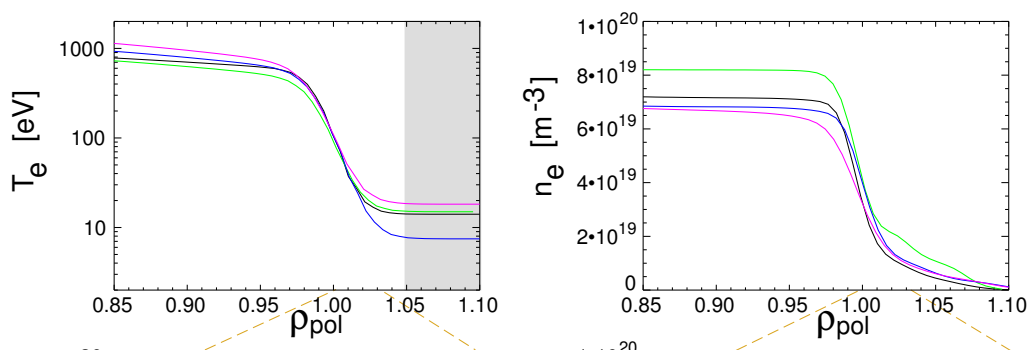

$\mathrm{dt}_{\mathrm{ELM}}=3-10 \mathrm{~ms}$
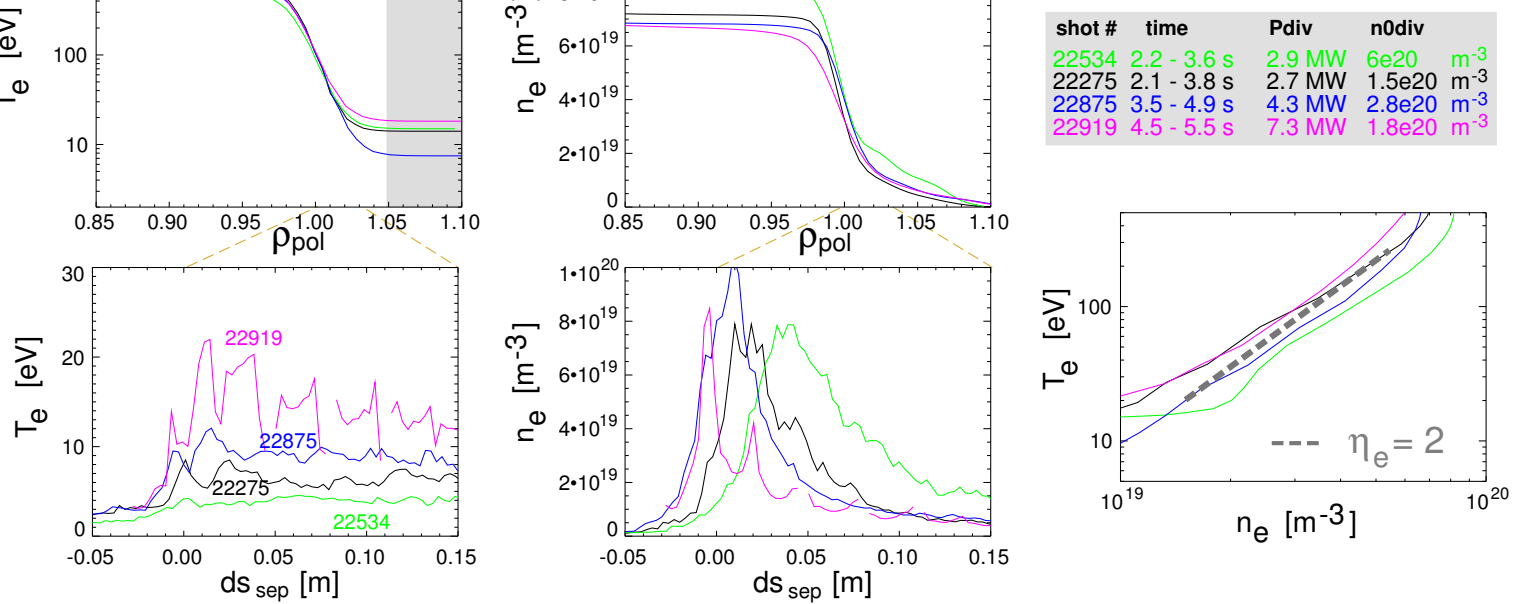

Fig. 3. Midplane and divertor profiles of $\mathrm{n}_{e}$ and $\mathrm{T}_{e}$ for a set of 4 discharges with different divertor densities and power fluxes. The $0.15 \mathrm{~m}$ from the strike point towards the SOL along the target correspond to about $18 \mathrm{~mm}$ in the outer midplane. The shaded region of the upstream $\mathrm{T}_{e}$ profiles marks the extrapolation of Thomson scattering data by the modified tanh fit. Also shown is the parameter $\eta_{e}$.
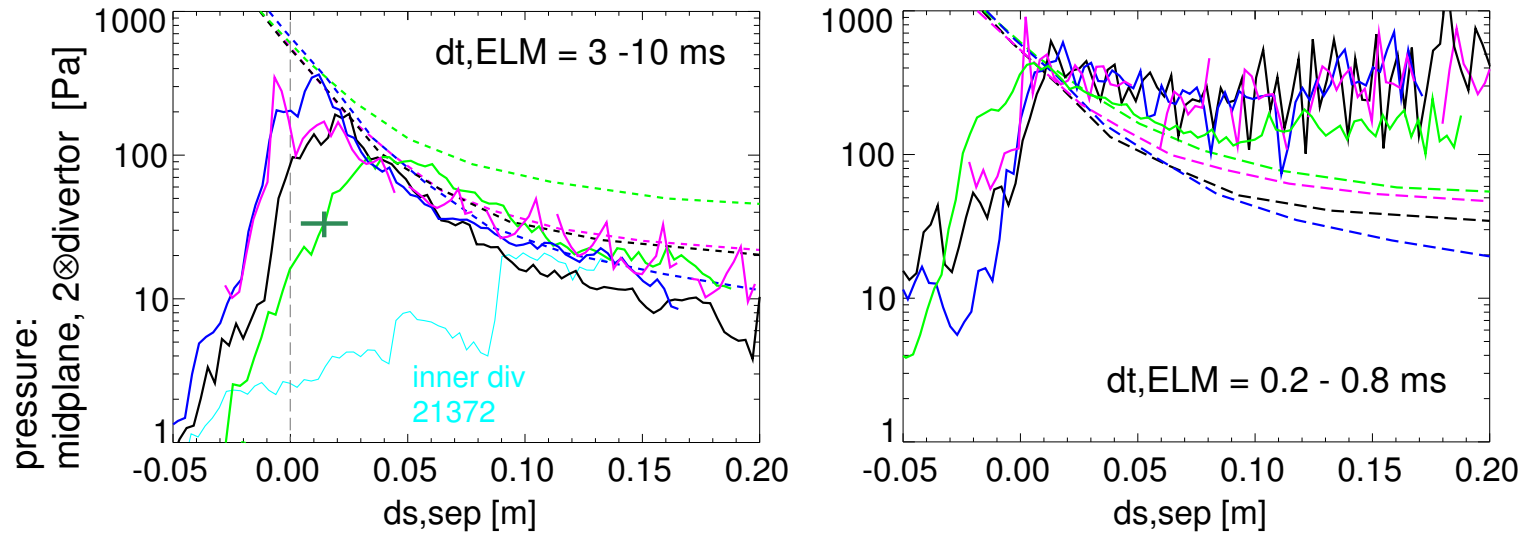

Fig. 4. Electron pressure in the outer midplane mapped to the divertor target (dashed lines) versus 2 $\times$ the divertor electron pressure from Langmuir probe measurements. Left: inter-ELM, right: during ELM. The inter-ELM pressure for the inner divertor plotted in light blue has been taken from a similar pulse of the previous campaign. 